International Journal of Modern Physics D

Vol. 14, No. 5 (2005) 797

World Scientific Publishing Company

\title{
Lie symmetries of the energy-momentum tensor for plane symmetric static spacetimes
}

\author{
K. Saifullah \\ Department of Mathematics, Quaid-i-Azam University, Islamabad, Pakistan \\ Electronic address: saifullah@qau.edu.pk
}

\begin{abstract}
Matter collineations (MCs) are the vector fields along which the energymomentum tensor remains invariant under the Lie transport. Invariance of the metric, the Ricci and the Riemann tensors have been studied extensively and the vectors along which these tensors remain invariant are called Killing vectors (KVs), Ricci collineations ( $\mathrm{RCs}$ ) and curvature collineations (CCs), respectively. In this paper plane symmetric static spacetimes have been studied for their MCs. Explicit form of MCs together with the Lie algebra admitted by them has been presented. Examples of spacetimes have been constructed for which MCs have been compared with their RCs and KVs. The comparison shows that neither of the sets of RCs and MCs contains the other, in general.
\end{abstract}




\section{Introduction}

One of the principal applications of Lie derivatives in theoretical physics is to express the notion that a tensor field is invariant under some transformation. We say that a tensor field $\mathbf{T}$ is invariant under a vector field $\mathbf{V}$ if

$$
£_{\mathbf{V}} \mathbf{T}=0,
$$

where $£_{\mathbf{V}}$ denotes Lie differentiation with respect to the vector $\mathbf{V}$. If $\mathbf{T}$ has physical importance then those special vector fields under which $\mathbf{T}$ is invariant will also be important. The manifolds of interest in theoretical physics have metrics, and it is therefore of considerable interest whenever the metric is invariant with respect to some vector field. These vector fields are called the Killing vectors (KVs) or isometries. After the spacetime metric, the curvature, the Ricci and the energy-momentum tensors are other important candidates which play a significant role in understanding the geometric structure and physical properties of spacetimes in relativity. While the isometries, provide information of the symmetries inherent in the spacetime, the symmetries of the matter-energy field are provided by MCs, vector fields along which the energy momentum tensor is invariant under the Lie transport. These symmetry properties are described by continuous groups of motions or collineations and they lead to conservation laws. For an introduction to spacetime symmetries and their significance the reader may see References $[1,2,3]$.

Formally, the KV is defined as follows. A manifold $M$ is said to admit a $\mathrm{KV}$ (or motion) $\xi^{a}$ if the Lie derivative of the metric $g_{a b}$ with respect to $\xi^{a}$ is conserved, i.e.

$$
£_{\xi} g_{a b}=0 .
$$

The vector $\xi^{a}$ is a Ricci collineation (RC) if the Lie derivative of the Ricci tensor, $R_{a b}$, with respect to. $\xi^{a}$ is conserved, i.e.

$$
£_{\xi} R_{a b}=0 .
$$

Since the Ricci tensor is built from the metric tensor, it must inherit its symmetries. Thus if the Lie derivative of $g_{a b}$ vanishes, it must vanish for $R_{a b}$ also. Hence every $\mathrm{KV}$ is an $\mathrm{RC}$ but the converse may not be true. The RCs which are not KVs are called proper RCs [4]. For plane symmetric spacetimes the RCs are finite if the Ricci tensor is non-degenerate; for degenerate case the RCs may be finite as well as infinite dimensional [4, 5]. If $R_{a b}$ in Eq. (1.3) is replaced by the energy-momentum tensor, $T_{a b}$, then the vector $\xi$ is called an MC. In component form this can be expressed as

$$
\xi^{c} T_{a b, c}+T_{a c} \xi_{, b}^{c}+T_{b c} \xi_{, a}^{c}=0
$$


Clearly, every $\mathrm{KV}$ is an MC also but the converse is not true. Recently, MCs for different spacetimes have been discussed in the literature [3, 6]. However, very little is known on this important subject, particularly, the relationship between RCs and MCs and there is a need for more research [7]. In this paper we study the Lie symmetries of the energy-momentum tensor, called the matter collineations (MCs), for plane symmetric static spacetimes, and compare them with their KVs and RCs $[4,5,8]$.

The plan of the paper is as follows. In Sections 2 and 3 we construct and solve the MC equations. Section 5 contains the algebra of the MCs obtained in the previous section. Examples of the metrics are given in Section 6, where MCs are compared with their RCs and KVs. Concluding remarks are given at the end.

\section{The matter collineation equations}

We take $\left(x^{0} x^{1} x^{2} x^{3}\right)=(t, x, y, z)$, so that, the most general plane symmetric static line element can be written as [9]

$$
d s^{2}=e^{\nu(x)} d t^{2}-d x^{2}-e^{\mu(x)}\left(d y^{2}+d z^{2}\right),
$$

where $\nu$ and $\mu$ are functions of $x$ only. For this metric the non-vanishing components of $T_{a b}$ are

$$
\begin{aligned}
& T_{00}=-\frac{e^{\nu(x)}}{4}\left(4 \mu^{\prime \prime}+3 \mu^{\prime^{2}}\right), \\
& T_{11}=\frac{1}{4}\left(2 \nu^{\prime} \mu^{\prime}+\mu^{\prime^{2}}\right), \\
& T_{22}=\frac{e^{\mu(x)}}{4}\left(2 \nu^{\prime \prime}+2 \mu^{\prime \prime}+\nu^{\prime^{2}}+\mu^{\prime^{2}}+\nu^{\prime} \mu^{\prime}\right)=T_{33},
\end{aligned}
$$

and those of the Ricci tensor are

$$
\begin{aligned}
& R_{00}=\frac{e^{\nu}}{4}\left(2 \nu^{\prime \prime}+\nu^{\prime 2}+2 \nu^{\prime} \mu^{\prime}\right), \\
& R_{11}=-\left(\frac{\nu^{\prime \prime}}{2}+\mu^{\prime \prime}+\frac{\nu^{\prime}}{4}+\frac{\mu^{\prime}}{2}\right), \\
& R_{22}=-\frac{e^{\mu}}{4}\left(2 \mu^{\prime \prime}+\nu^{\prime} \mu^{\prime}+2 \mu^{\prime^{2}}\right)=R_{33} .
\end{aligned}
$$

Here $^{\prime \prime}$ denotes differentiation with respect to $x$. The Ricci scalar is given by

$$
R=\nu^{\prime \prime}+2 \mu^{\prime \prime}+\frac{1}{2}\left(\nu^{\prime 2}+3 \mu^{\prime^{2}}+2 \nu^{\prime} \mu^{\prime}\right)
$$

Writing $T_{i i}=T_{i}$, for $i=0,1,2,3$, the MC equations Eq. (1.4) for the MC vector $\xi$ take the form 


$$
\begin{aligned}
T_{0}^{\prime} \xi^{1}+2 T_{0} \xi_{, 0}^{0} & =0, \\
T_{0} \xi_{, 1}^{0}+T_{1} \xi_{, 0}^{1} & =0, \\
T_{0} \xi_{, 2}^{0}+T_{2} \xi_{, 0}^{2} & =0, \\
T_{0} \xi_{, 3}^{0}+T_{2} \xi_{, 0}^{3} & =0, \\
T_{1}^{\prime} \xi^{1}+2 T_{1} \xi_{, 1}^{1} & =0, \\
T_{1} \xi_{, 2}^{1}+T_{2} \xi_{, 1}^{2} & =0, \\
T_{1} \xi_{, 3}^{1}+T_{2} \xi_{, 1}^{3} & =0, \\
T_{2}^{\prime} \xi^{1}+2 T_{2} \xi_{, 2}^{2} & =0, \\
T_{2}\left(\xi_{, 3}^{2}+\xi_{, 2}^{3}\right) & =0, \\
T_{2}^{\prime} \xi^{1}+2 T_{2} \xi_{, 3}^{3} & =0 .
\end{aligned}
$$

These are ten non-linear coupled partial differential equations for $\xi^{0}, \xi^{1}, \xi^{2}, \xi^{3}$, and $T_{0}, T_{1}, T_{2}, T_{3}$. The $\xi^{i}$ depend on $t, x, y$ and $z$; and the $T_{i}$ on $x$ only.

\section{Solution of the matter collineation equations}

Solving Eqs. (2.5)- (2.14) simultaneously we obtain the components of the MC vector, $\xi$. Since the procedure for the solution of similar systems of partial differential equations has appeared in the literature $[4,10]$, we do not give the calculations and only present the results. The procedure, roughly speaking, is as follows. We first integrate any of these equations to obtain the components of $\xi$ in terms of arbitrary functions of the coordinates. Using this form of $\xi$ in other $\mathrm{MC}$ equations will give conditions on these arbitrary functions. Going back and forth in this way and checking consistency with the MC equations at every step until these functions are determined explicitly yields the final form of $\xi$ involving arbitrary constants. In the course of finding these solutions we get constraints on the components of $\mathbf{T}$. Thus we will arrive at various cases of MCs corresponding to these constraints. Solving these constraints, which are often differential in nature, gives the spacetimes. Now, we list these cases.

Case 1

Constraints on $T_{a b}$ :

$$
T_{0}^{\prime} \neq 0,\left(\frac{T_{2}^{\prime}}{T_{2} \sqrt{T_{1}}}\right)^{\prime} \neq 0,\left(\frac{T_{0}}{T_{2}}\right)^{\prime}=0 .
$$


MCs: Let $\frac{T_{0}}{T_{2}}=k$.

$$
\begin{aligned}
& \xi^{0}=-\frac{1}{k}\left(c_{6} z+c_{5} y\right)+c_{1}, \\
& \xi^{1}=0 \\
& \xi^{2}=c_{4} z+k c_{5} t+c_{2}, \\
& \xi^{3}=-y c_{4}+k c_{6} t+c_{3} .
\end{aligned}
$$

Case 2

Constraints on $T_{a b}$ :

$$
T_{0}^{\prime} \neq 0,\left(\frac{T_{2}^{\prime}}{T_{2} \sqrt{T_{1}}}\right)^{\prime} \neq 0,\left(\frac{T_{0}}{T_{2}}\right)^{\prime} \neq 0
$$

MCs:

$$
\begin{aligned}
& \xi^{0}=c_{1}, \\
& \xi^{1}=0, \\
& \xi^{2}=c_{4} z+c_{2}, \\
& \xi^{3}=-c_{4} y+c_{3} .
\end{aligned}
$$

Case 3

Constraints on $T_{a b}$ :

$$
T_{0}^{\prime} \neq 0,\left(\frac{T_{2}^{\prime}}{T_{2} \sqrt{T_{1}}}\right)^{\prime}=0, T_{2}^{\prime} \neq 0,\left(\frac{T_{2}}{T_{0}}\right)^{\prime} \neq 0,\left(\frac{T_{0}^{\prime}}{T_{0} \sqrt{T_{1}}}\right)^{\prime} \neq 0 .
$$

MCs:

$$
\begin{aligned}
& \xi^{0}=c_{1}, \\
& \xi^{1}=0, \\
& \xi^{2}=c_{4} z+c_{2}, \\
& \xi^{3}=-c_{4} y+c_{3} .
\end{aligned}
$$

Case 4

Constraints on $T_{a b}$ :

$$
T_{0}^{\prime} \neq 0,\left(\frac{T_{2}^{\prime}}{T_{2} \sqrt{T_{1}}}\right)^{\prime}=0, T_{2}^{\prime} \neq 0,\left(\frac{T_{2}}{T_{0}}\right)^{\prime} \neq 0,\left(\frac{T_{0}^{\prime}}{T_{0} \sqrt{T_{1}}}\right)^{\prime}=0 .
$$

MCs: Let $\frac{R_{2}^{\prime}}{R_{2} \sqrt{R_{1}}}=\alpha, \frac{T_{0}^{\prime}}{T_{0} \sqrt{T_{1}}}=\beta \neq \alpha$, where $\alpha$ and $\beta$ are constants.

$$
\begin{aligned}
& \xi^{0}=\frac{\beta}{\alpha} c_{5} t+c_{1}, \\
& \xi^{1}=-c_{5} \frac{2}{\alpha \sqrt{T_{1}}}, \\
& \xi^{2}=c_{4} z+c_{5} y+c_{2}, \\
& \xi^{3}=-c_{4} y+c_{5} z+c_{3} .
\end{aligned}
$$




\section{Case 5}

Constraints on $T_{a b}$ :

$$
T_{0}^{\prime} \neq 0,\left(\frac{T_{2}^{\prime}}{T_{2} \sqrt{T_{1}}}\right)^{\prime}=0, T_{2}^{\prime} \neq 0,\left(\frac{T_{2}}{T_{0}}\right)^{\prime}=0 .
$$

MCs: Here we write $\frac{R_{2}^{\prime}}{R_{2} \sqrt{R_{1}}}=\alpha, T_{2}=-\delta T_{0}$.

$\xi^{0}=\frac{c_{7}}{2}\left(t^{2}-\frac{4}{\alpha^{2} T_{0}}+\delta y^{2}+\delta z^{2}\right)+c_{6} \delta z-c_{8} y t+c_{9} t z+c_{5} \delta y+c_{10} t+c_{1}$,

$\xi^{1}=-\frac{2}{\alpha \sqrt{T}_{1}}\left(c_{7} t-c_{8} y+c_{9} z+c_{10}\right)$,

$\xi^{2}=c_{7} y t+\frac{c_{8}}{2}\left(-\frac{t^{2}}{\delta}+\frac{4}{\alpha^{2} T_{2}}-y^{2}+z^{2}\right)+c_{5} t+c_{9} y z+c_{10} y+c_{4} z+c_{2}$,

$\xi^{3}=c_{7} z t-c_{8} y z-\frac{c_{9}}{2}\left(-\frac{t^{2}}{\delta}+\frac{4}{\alpha^{2} T_{2}}+y^{2}-z^{2}\right)+c_{6} t+c_{10} z-c_{4} y+c_{3}$.

Case 6

\section{Constraints on $T_{a b}$ :}

$$
T_{0}^{\prime} \neq 0, T_{2}^{\prime}=0,\left(\frac{\left(\sqrt{T_{0}}\right)^{\prime}}{\sqrt{T_{1}}}\right)^{\prime}=0 .
$$

MCs: Put $\frac{\left(\sqrt{T_{0}}\right)^{\prime}}{\sqrt{T_{1}}}=\gamma$ a constant.

$$
\begin{aligned}
& \xi^{0}=\frac{1}{\sqrt{T_{0}}}\left[z\left(c_{7} \sin \gamma t-c_{8} \cos \gamma t\right)+y\left(c_{5} \sin \gamma t-c_{6} \cos \gamma t\right)\right. \\
& \left.-\left(c_{9} \sin \gamma t-c_{10} \cos \gamma t\right)\right]+c_{1}, \\
& \xi^{1}=-\frac{1}{\sqrt{T_{1}}}\left[z\left(c_{7} \cos \gamma t+c_{8} \sin \gamma t\right)+y\left(c_{5} \cos \gamma t+c_{6} \sin \gamma t\right)\right. \\
& \left.-\left(c_{9} \cos \gamma t+c_{10} \sin \gamma t\right)\right], \\
& \xi^{2}=\frac{\sqrt{T_{0}}}{\gamma T_{2}}\left(c_{5} \cos \gamma t+c_{6} \sin \gamma t\right)+c_{4} z+c_{2}, \\
& \xi^{3}=\frac{\sqrt{T_{0}}}{\gamma T_{2}}\left(c_{7} \cos \gamma t+c_{8} \sin \gamma t\right)-c_{4} y+c_{3} .
\end{aligned}
$$

\section{Case 7}

\section{Constraints on $T_{a b}$ :}

$$
T_{0}^{\prime} \neq 0, T_{2}^{\prime}=0,\left(\frac{\left(\sqrt{T_{0}}\right)^{\prime}}{\sqrt{T_{1}}}\right)^{\prime} \neq 0,\left[\frac{T_{0}}{2 \sqrt{T_{1}}}\left(\frac{T_{0}^{\prime}}{T_{0} \sqrt{T_{1}}}\right)^{\prime}\right]^{\prime}=0 .
$$

MCs: This implies that $\frac{T_{0}^{\prime}}{T_{0} \sqrt{T_{1}}}=$ constant $=\lambda \neq 0$.

$$
\begin{aligned}
& \xi^{0}=c_{5}\left(\frac{1}{\lambda T_{0}}-\frac{\lambda}{4} t^{2}\right)-c_{6} \frac{\lambda}{2} t+c_{1}, \\
& \xi^{1}=\frac{1}{\sqrt{T_{1}}}\left(c_{5} t+c_{6}\right), \\
& \xi^{2}=c_{4} z+c_{2}, \\
& \xi^{3}=-c_{4} y+c_{3} .
\end{aligned}
$$


Case 8

Constraints on $T_{a b}$ :

$$
T_{0}^{\prime} \neq 0, T_{2}^{\prime}=0,\left(\frac{\left(\sqrt{T_{0}}\right)^{\prime}}{\sqrt{T_{1}}}\right)^{\prime} \neq 0,\left[\frac{T_{0}}{\sqrt{T_{1}}}\left(\frac{T_{0}^{\prime}}{T_{0} \sqrt{T_{1}}}\right)^{\prime}\right]^{\prime} \neq 0 .
$$

MCs:

$$
\begin{aligned}
& \xi^{0}=c_{1}, \\
& \xi^{1}=0, \\
& \xi^{2}=c_{4} z+c_{2}, \\
& \xi^{3}=-c_{4} y+c_{3} .
\end{aligned}
$$

Case 9

Constraints on $T_{a b}$ :

$$
T_{0}^{\prime}=0, T_{2}^{\prime}=0 .
$$

MCs: We put $T_{0}=-\alpha$ and $T_{2}=\beta$.

$$
\begin{aligned}
& \xi^{0}=-\frac{c_{6}}{\alpha} \int \sqrt{T_{1}} d x+c_{7} y+c_{8} z+c_{1}, \\
& \xi^{1}=\frac{1}{\sqrt{T_{1}}}\left(c_{6} t+c_{9} y+c_{10} z+c_{5}\right), \\
& \xi^{2}=\frac{\alpha}{\beta} c_{7} t-\frac{1}{\beta} c_{9} \int \sqrt{T_{1}} d x+c_{4} z+c_{2}, \\
& \xi^{3}=\frac{\alpha}{\beta} c_{8} t-\frac{1}{\beta} c_{10} \int \sqrt{T_{1}} d x-\beta c_{4} y+c_{3} .
\end{aligned}
$$

Case 10

Constraints on $T_{a b}$ :

$$
T_{2}^{\prime} \neq 0,\left[\frac{T_{2}}{\sqrt{T_{1}}}\left(\frac{T_{2}^{\prime}}{T_{2} \sqrt{T_{1}}}\right)^{\prime}\right]^{\prime} \neq 0
$$

MCs:

$$
\begin{aligned}
& \xi^{0}=c_{1}, \\
& \xi^{1}=0 \\
& \xi^{2}=c_{4} z+c_{2}, \\
& \xi^{3}=-c_{4} y+c_{3} .
\end{aligned}
$$

Case 11

Constraints on $T_{a b}$ :

$$
T_{2}^{\prime} \neq 0,\left[\frac{T_{2}}{\sqrt{T_{1}}}\left(\frac{T_{2}^{\prime}}{2 T_{2} \sqrt{T_{1}}}\right)^{\prime}\right]^{\prime}=0,\left(\frac{T_{2}^{\prime}}{T_{2} \sqrt{T_{1}}}\right)^{\prime} \neq 0 .
$$


MCs:

$$
\begin{aligned}
& \xi^{0}=c_{1}, \\
& \xi^{1}=0, \\
& \xi^{2}=c_{4} z+c_{2}, \\
& \xi^{3}=-c_{4} y+c_{3} .
\end{aligned}
$$

Case 12

Constraints on $T_{a b}$ :

$$
T_{0}^{\prime}=0, T_{2}^{\prime} \neq 0,\left(\frac{T_{2}^{\prime}}{T_{2} \sqrt{T_{1}}}\right)^{\prime}=0 .
$$

MCs: Put $\frac{T_{2}^{\prime}}{T_{2} \sqrt{T_{1}}}=k_{1}$

$$
\begin{aligned}
& \xi^{0}=c_{1}, \\
& \xi^{1}=\frac{1}{\sqrt{T_{1}}}\left(c_{6} y+c_{7} z+c_{5}\right), \\
& \xi^{2}=-c_{6}\left(\int \frac{\sqrt{T_{1}}}{T_{2}} d x+k_{1} \frac{y^{2}}{2}-k_{1} \frac{z^{2}}{2}\right)-k_{1} c_{7} y z-k_{1} c_{5} y-c_{4} z+c_{2}, \\
& \xi^{3}=-k_{1} c_{6} y z-c_{7}\left(\int \frac{\sqrt{T_{1}}}{T_{2}} d x-\frac{k_{1}}{2} y^{2}+k_{1} \frac{z^{2}}{2}\right)-k_{1} c_{5} z+c_{4} y+c_{3} .
\end{aligned}
$$

For the degenerate $T_{a b}$, i.e. when $\operatorname{det}\left(T_{a b}\right)=0$, we get MCs admitting infinite dimensional Lie algebras, except in one case when $T_{1}=0, T_{i} \neq 0$ for $i=0,2,3$. Cases 13 and 14 have degenerate energy-momentum tensor but admit finite MCs.

Case 13

Constraints on $T_{a b}$ :

$$
T_{1}=0, T_{0} \neq 0, T_{2} \neq 0,\left(\frac{T_{0}^{\prime} T_{2}}{T_{0} T_{2}^{\prime}}\right)^{\prime}=0 .
$$

MCs: Put $\frac{T_{0}^{\prime} T_{2}}{T_{0} T_{2}^{\prime}}=k_{2}$.

$$
\begin{aligned}
& \xi^{0}=c_{5} t+c_{1}, \\
& \xi^{1}=-c_{5} \frac{2 T_{0}}{T_{0}^{\prime}}, \\
& \xi^{2}=c_{4} z+\frac{1}{k_{2}} c_{5} y+c_{2}, \\
& \xi^{3}=-c_{4} y+\frac{1}{k_{2}} c_{5} z+c_{3} .
\end{aligned}
$$

Case 14

\section{Constraints on $T_{a b}$ :}

$$
T_{1}=0, T_{0} \neq 0, T_{2} \neq 0, T_{0}^{\prime} \neq 0, T_{2}^{\prime} \neq 0,\left(\frac{T_{0}}{T_{2}}\right)^{\prime}=0 .
$$


MCs: Here we write $T_{0}=-\alpha T_{2}$.

$$
\begin{aligned}
& \xi^{0}=c_{7}\left(\frac{y^{2}}{2}+\frac{z^{2}}{2}+\alpha \frac{t^{2}}{2}\right)+c_{8} t y+c_{5} y+c_{9} t z+c_{6} z+c_{10} t+c_{1}, \\
& \xi^{1}=-\frac{2 T_{0}}{T_{0}^{\prime}}\left(\alpha c_{7} t+c_{8} y+c_{9} z+c_{10}\right), \\
& \xi^{2}=\alpha c_{7} t y+c_{8}\left(\alpha \frac{t^{2}}{2}+\frac{y^{2}}{2}-\frac{z^{2}}{2}\right)+\alpha c_{5} t+c_{9} y z+c_{10} y-c_{4} z+c_{2}, \\
& \xi^{3}=\alpha c_{7} t z+c_{8} y z+c_{9}\left(\alpha \frac{t^{2}}{2}-\frac{y^{2}}{2}+\frac{z^{2}}{2}\right)+\alpha c_{6} t+c_{10} z+c_{4} y+c_{3} .
\end{aligned}
$$

Case 15

If either $T_{0}$ or $T_{2}$ (or both) are zero $T_{a b}$ becomes degenerate and MCs admit infinite dimensional Lie algebra.

\section{Lie algebras of matter collineations}

If a set of vector fields on a manifold under the operation of Lie bracket (defined by the Lie derivative on a manifold) satisfies the conditions of anti-commutativity and Jacobi's identity, one gets a Lie algebra. Here we provide the Lie algebraic structure for the MC vector fields obtained in the last section and identify their nature. We also classify them into solvable and semisimple algebras and identify some of their sub-algebras.

\section{Case 1}

Generators:

$$
\begin{aligned}
& \mathbf{X}_{1}=\partial_{t} \\
& \mathbf{X}_{2}=\partial_{y} \\
& \mathbf{X}_{3}=\partial_{z} \\
& \mathbf{X}_{4}=z \partial_{y}-y \partial_{z} \\
& \mathbf{X}_{5}=y \partial_{t}+k^{2} t \partial_{y} \\
& \mathbf{X}_{6}=z \partial_{t}+k^{2} t \partial_{z}
\end{aligned}
$$

Algebra:

$$
\begin{array}{lll}
{\left[\mathbf{X}_{1}, \mathbf{X}_{5}\right]=k^{2} \mathbf{X}_{2},} & {\left[\mathbf{X}_{1}, \mathbf{X}_{6}\right]=k^{2} \mathbf{X}_{3},} & {\left[\mathbf{X}_{2}, \mathbf{X}_{4}\right]=-\mathbf{X}_{3},} \\
{\left[\mathbf{X}_{2}, \mathbf{X}_{5}\right]=\mathbf{X}_{1},} & {\left[\mathbf{X}_{3}, \mathbf{X}_{4}\right]=\mathbf{X}_{2},} & {\left[\mathbf{X}_{3}, \mathbf{X}_{6}\right]=\mathbf{X}_{1},} \\
{\left[\mathbf{X}_{4}, \mathbf{X}_{5}\right]=\mathbf{X}_{6},} & {\left[\mathbf{X}_{4}, \mathbf{X}_{6}\right]=-\mathbf{X}_{5},} & {\left[\mathbf{X}_{5}, \mathbf{X}_{6}\right]=-k^{2} \mathbf{X}_{4},} \\
{\left[\mathbf{X}_{i}, \mathbf{X}_{j}\right]=0, \text { otherwise. }} &
\end{array}
$$

This is $S O(1,2) \times\left[S O(2) \otimes \mathbb{R}^{2}\right]$ where ' $\times$ ' represents the semi-direct and ' $\otimes$ ' the direct product. Here $\mathbf{X}_{5}$ and $\mathbf{X}_{6}$ are the Lorentz boosts in $y$ and $z$ directions. 
$\mathbf{X}_{4}$ is a rotation in $y$ and $z$. This is a semisimple algebra having $\left\langle\mathbf{X}_{4}, \mathbf{X}_{5}, \mathbf{X}_{6}\right\rangle$ as a subalgebra.

\section{Case 2}

Generators:

$$
\begin{aligned}
& \mathbf{X}_{1}=\partial_{t}, \\
& \mathbf{X}_{2}=\partial_{y}, \\
& \mathbf{X}_{3}=\partial_{z}, \\
& \mathbf{X}_{4}=z \partial_{y}-y \partial_{z} .
\end{aligned}
$$

Algebra:

$$
\left[\mathbf{X}_{2}, \mathbf{X}_{4}\right]=-\mathbf{X}_{3}, \quad\left[\mathbf{X}_{3}, \mathbf{X}_{4}\right]=\mathbf{X}_{2}, \quad\left[\mathbf{X}_{i}, \mathbf{X}_{j}\right]=0, \text { otherwise }
$$

This can be written as $\{S O(2) \times[\mathbb{R} \otimes S O(2)]\} \otimes \mathbb{R}$ and is solvable.

\section{Case 3}

Generators:

$$
\begin{aligned}
& \mathbf{X}_{1}=\partial_{t}, \\
& \mathbf{X}_{2}=\partial_{y}, \\
& \mathbf{X}_{3}=\partial_{z}, \\
& \mathbf{X}_{4}=z \partial_{y}-y \partial_{z} .
\end{aligned}
$$

Algebra:

$$
\left[\mathbf{X}_{2}, \mathbf{X}_{4}\right]=-\mathbf{X}_{3}, \quad\left[\mathbf{X}_{3}, \mathbf{X}_{4}\right]=\mathbf{X}_{2}, \quad\left[\mathbf{X}_{i}, \mathbf{X}_{j}\right]=0, \text { otherwise }
$$

This is the same as the previous case.

Case 4

Generators:

$$
\begin{aligned}
& \mathbf{X}_{1}=\partial_{t}, \\
& \mathbf{X}_{2}=\partial_{y}, \\
& \mathbf{X}_{3}=\partial_{z}, \\
& \mathbf{X}_{4}=z \partial_{y}-y \partial_{z}, \\
& \mathbf{X}_{5}=\frac{\beta}{\alpha} t \partial_{t}-\frac{2}{\alpha \sqrt{T_{1}}} \partial_{x}+y \partial_{y}+z \partial_{z}
\end{aligned}
$$

Algebra:

$$
\begin{array}{lll}
{\left[\mathbf{X}_{1}, \mathbf{X}_{5}\right]=\frac{\beta}{\alpha} \mathbf{X}_{1},} & {\left[\mathbf{X}_{2}, \mathbf{X}_{4}\right]=-\mathbf{X}_{3},} & {\left[\mathbf{X}_{2}, \mathbf{X}_{5}\right]=\mathbf{X}_{2},} \\
{\left[\mathbf{X}_{3}, \mathbf{X}_{4}\right]=\mathbf{X}_{2},} & {\left[\mathbf{X}_{3}, \mathbf{X}_{5}\right]=\mathbf{X}_{3},} & {\left[\mathbf{X}_{i}, \mathbf{X}_{j}\right]=0, \text { otherwise }}
\end{array}
$$

This is a solvable algebra which can be written as $G_{5}=\left\langle G_{4}, \mathbf{X}_{5}\right\rangle$, where 
$G_{4}=\{S O(2) \times[\mathbb{R} \otimes S O(2)]\} \otimes \mathbb{R}$.

Case 5

Generators:

$$
\begin{aligned}
& \mathbf{X}_{1}=\partial_{t} \\
& \mathbf{X}_{2}=\partial_{y} \\
& \mathbf{X}_{3}=\partial_{z} \\
& \mathbf{X}_{4}=z \partial_{y}-y \partial_{z}, \\
& \mathbf{X}_{5}=\delta y \partial_{t}+t \partial_{y}, \\
& \mathbf{X}_{6}=\delta z \partial_{t}+t \partial_{z} \\
& \mathbf{X}_{7}=\frac{1}{2}\left(t^{2}-\frac{4}{\alpha^{2} T_{0}}+\delta y^{2}+\delta z^{2}\right) \partial_{t}-\frac{2}{\alpha \sqrt{T_{1}}} t \partial_{x}+y t \partial_{y}+z t \partial_{z}, \\
& \mathbf{X}_{8}=-y t \partial_{t}+\frac{2}{\alpha \sqrt{T_{1}}} y \partial_{x}+\frac{1}{2}\left(-\frac{t^{2}}{\delta}+\frac{4}{\alpha^{2} T_{2}}-y^{2}+z^{2}\right) \partial_{y}-y z \partial_{z}, \\
& \mathbf{X}_{9}=t z \partial_{t}-\frac{2}{\alpha \sqrt{T_{1}}} z \partial_{x}+y z \partial_{y}-\frac{1}{2}\left(-\frac{t^{2}}{\delta}+\frac{4}{\alpha^{2} T_{2}}+y^{2}-z^{2}\right) \partial_{z}, \\
& \mathbf{X}_{10}=t \partial_{t}-\frac{2}{\alpha \sqrt{T_{1}}} \partial_{x}+y \partial_{y}+z \partial_{z} .
\end{aligned}
$$

Algebra:

$\begin{array}{lll}{\left[\mathbf{X}_{1}, \mathbf{X}_{5}\right]=\mathbf{X}_{2},} & {\left[\mathbf{X}_{1}, \mathbf{X}_{6}\right]=\mathbf{X}_{3},} & {\left[\mathbf{X}_{1}, \mathbf{X}_{7}\right]=\mathbf{X}_{10},} \\ {\left[\mathbf{X}_{1}, \mathbf{X}_{8}\right]=\frac{1}{\delta} \mathbf{X}_{5},} & {\left[\mathbf{X}_{1}, \mathbf{X}_{9}\right]=\frac{1}{\delta} \mathbf{X}_{6},} & {\left[\mathbf{X}_{1}, \mathbf{X}_{10}\right]=\mathbf{X}_{1},} \\ {\left[\mathbf{X}_{2}, \mathbf{X}_{4}\right]=-\mathbf{X}_{3},} & {\left[\mathbf{X}_{2}, \mathbf{X}_{5}\right]=\delta \mathbf{X}_{1},} & {\left[\mathbf{X}_{2}, \mathbf{X}_{7}\right]=\mathbf{X}_{5},} \\ {\left[\mathbf{X}_{2}, \mathbf{X}_{8}\right]=-\mathbf{X}_{10}} & {\left[\mathbf{X}_{2}, \mathbf{X}_{9}\right]=\mathbf{X}_{4},} & {\left[\mathbf{X}_{2}, \mathbf{X}_{10}\right]=\mathbf{X}_{2},} \\ {\left[\mathbf{X}_{3}, \mathbf{X}_{4}\right]=\mathbf{X}_{2},} & {\left[\mathbf{X}_{3}, \mathbf{X}_{6}\right]=\delta \mathbf{X}_{1},} & {\left[\mathbf{X}_{3}, \mathbf{X}_{7}\right]=\mathbf{X}_{6},} \\ {\left[\mathbf{X}_{3}, \mathbf{X}_{8}\right]=\mathbf{X}_{4},} & {\left[\mathbf{X}_{3}, \mathbf{X}_{9}\right]=\mathbf{X}_{10},} & {\left[\mathbf{X}_{3}, \mathbf{X}_{10}\right]=\mathbf{X}_{3},} \\ {\left[\mathbf{X}_{4}, \mathbf{X}_{5}\right]=\mathbf{X}_{6},} & {\left[\mathbf{X}_{4}, \mathbf{X}_{6}\right]=-\mathbf{X}_{5},} & {\left[\mathbf{X}_{4}, \mathbf{X}_{8}\right]=-\mathbf{X}_{9},} \\ {\left[\mathbf{X}_{4}, \mathbf{X}_{9}\right]=\mathbf{X}_{8},} & {\left[\mathbf{X}_{5}, \mathbf{X}_{6}\right]=-\delta \mathbf{X}_{4},} & {\left[\mathbf{X}_{5}, \mathbf{X}_{7}\right]=-\delta \mathbf{X}_{8},} \\ {\left[\mathbf{X}_{5}, \mathbf{X}_{8}\right]=-\mathbf{X}_{7},} & {\left[\mathbf{X}_{6}, \mathbf{X}_{7}\right]=\delta \mathbf{X}_{9},} & {\left[\mathbf{X}_{6}, \mathbf{X}_{9}\right]=\mathbf{X}_{7},} \\ {\left[\mathbf{X}_{7}, \mathbf{X}_{10}\right]=-\mathbf{X}_{7},} & {\left[\mathbf{X}_{8}, \mathbf{X}_{10}\right]=-\mathbf{X}_{8},} & {\left[\mathbf{X}_{9}, \mathbf{X}_{10}\right]=-\mathbf{X}_{9},} \\ {\left[\mathbf{X}_{i}, \mathbf{X}_{j}\right]=0, \text { otherwise. }} & \end{array}$

This $S O(1,4)$ or $S O(2,3)$ is the maximal semisimple anti-de Sitter algebra. It has 3 dimensional subalgebras $\left\{\mathbf{X}_{4}, \mathbf{X}_{5}, \mathbf{X}_{6}\right\}$ of rotations and $\left\{\mathbf{X}_{8}, \mathbf{X}_{9}, \mathbf{X}_{10}\right\} ; 4$ dimensional subalgebras $\left\{\mathbf{X}_{1}, \mathbf{X}_{2}, \mathbf{X}_{3}, \mathbf{X}_{4}\right\}$ and $\left\{\mathbf{X}_{7}, \mathbf{X}_{8}, \mathbf{X}_{9}, \mathbf{X}_{10}\right\}$; and 6 dimensional subalgebras $\left\{\mathbf{X}_{1}, \mathbf{X}_{2}, \mathbf{X}_{3}, \mathbf{X}_{4}, \mathbf{X}_{5}, \mathbf{X}_{6}\right\}$ in it. 


\section{Case 6}

Generators:

$$
\begin{aligned}
& \mathbf{X}_{1}=\partial_{t}, \\
& \mathbf{X}_{2}=\partial_{y}, \\
& \mathbf{X}_{3}=\partial_{z}, \\
& \mathbf{X}_{4}=z \partial_{y}-y \partial_{z}, \\
& \mathbf{X}_{5}=\frac{1}{\sqrt{T_{0}}} y \sin \gamma t \partial_{t}-\frac{1}{\sqrt{T_{1}}} y \cos \gamma t \partial_{x}+\frac{\sqrt{T_{0}}}{\gamma T_{2}} \cos \gamma t \partial_{y}, \\
& \mathbf{X}_{6}=-\frac{1}{\sqrt{T_{0}}} y \cos \gamma t \partial_{t}-\frac{1}{\sqrt{T_{1}}} y \sin \gamma t \partial_{x}+\frac{\sqrt{T_{0}}}{\gamma T_{2}} \sin \gamma t \partial_{y}, \\
& \mathbf{X}_{7}=\frac{1}{\sqrt{T_{0}}} z \sin \gamma t \partial_{t}-\frac{1}{\sqrt{T_{1}}} z \cos \gamma t \partial_{x}+\frac{\sqrt{T_{0}}}{\gamma T_{2}} \cos \gamma t \partial_{z}, \\
& \mathbf{X}_{8}=-\frac{1}{\sqrt{T_{0}}} z \cos \gamma t \partial_{t}-\frac{1}{\sqrt{T_{1}}} z \sin \gamma t \partial_{x}+\frac{\sqrt{T_{0}}}{\gamma T_{2}} \sin \gamma t \partial_{z}, \\
& \mathbf{X}_{9}=-\frac{1}{\sqrt{T_{0}}} \sin \gamma t \partial_{t}+\frac{1}{\sqrt{T_{1}}} \cos \gamma t \partial_{x}, \\
& \mathbf{X}_{10}=\frac{1}{\sqrt{T_{0}}} \cos \gamma t \partial_{t}+\frac{1}{\sqrt{T_{1}}} \sin \gamma t \partial_{x} .
\end{aligned}
$$

Algebra:

$$
\begin{array}{lll}
{\left[\mathbf{X}_{1}, \mathbf{X}_{5}\right]=-\mathbf{X}_{6},} & {\left[\mathbf{X}_{1}, \mathbf{X}_{6}\right]=\mathbf{X}_{5},} & {\left[\mathbf{X}_{1}, \mathbf{X}_{7}\right]=-\mathbf{X}_{8},} \\
{\left[\mathbf{X}_{1}, \mathbf{X}_{8}\right]=\mathbf{X}_{7},} & {\left[\mathbf{X}_{1}, \mathbf{X}_{9}\right]=-\mathbf{X}_{10},} & {\left[\mathbf{X}_{1}, \mathbf{X}_{10}\right]=\mathbf{X}_{9},} \\
{\left[\mathbf{X}_{2}, \mathbf{X}_{4}\right]=-\mathbf{X}_{3},} & {\left[\mathbf{X}_{2}, \mathbf{X}_{5}\right]=-\mathbf{X}_{9},} & {\left[\mathbf{X}_{2}, \mathbf{X}_{6}\right]=-\mathbf{X}_{10},} \\
{\left[\mathbf{X}_{3}, \mathbf{X}_{4}\right]=\mathbf{X}_{2},} & {\left[\mathbf{X}_{3}, \mathbf{X}_{7}\right]=-\mathbf{X}_{9},} & {\left[\mathbf{X}_{3}, \mathbf{X}_{8}\right]=-\mathbf{X}_{10},} \\
{\left[\mathbf{X}_{4}, \mathbf{X}_{5}\right]=\mathbf{X}_{7},} & {\left[\mathbf{X}_{4}, \mathbf{X}_{6}\right]=\mathbf{X}_{8},} & {\left[\mathbf{X}_{4}, \mathbf{X}_{7}\right]=-\mathbf{X}_{5},} \\
{\left[\mathbf{X}_{4}, \mathbf{X}_{8}\right]=-\mathbf{X}_{6},} & {\left[\mathbf{X}_{5}, \mathbf{X}_{6}\right]=-\frac{1}{\gamma T_{2}} \mathbf{X}_{1},} & {\left[\mathbf{X}_{5}, \mathbf{X}_{7}\right]=\frac{1}{T_{2}} \mathbf{X}_{4},} \\
{\left[\mathbf{X}_{5}, \mathbf{X}_{9}\right]=-\frac{1}{T_{2}} \mathbf{X}_{2},} & {\left[\mathbf{X}_{6}, \mathbf{X}_{8}\right]=\frac{1}{T_{2}} \mathbf{X}_{4},} & {\left[\mathbf{X}_{6}, \mathbf{X}_{10}\right]=-\frac{1}{T_{2}} \mathbf{X}_{2},} \\
{\left[\mathbf{X}_{7}, \mathbf{X}_{8}\right]=-\frac{1}{\gamma T_{2}} \mathbf{X}_{1},} & {\left[\mathbf{X}_{7}, \mathbf{X}_{9}\right]=-\frac{1}{T_{2}} \mathbf{X}_{3},} & {\left[\mathbf{X}_{8}, \mathbf{X}_{10}\right]=-\frac{1}{T_{2}} \mathbf{X}_{3},} \\
{\left[\mathbf{X}_{i}, \mathbf{X}_{j}\right]=0, \text { otherwise. }} &
\end{array}
$$

This is again a 10 dimensional semisimple algebra and has $\left\{\mathbf{X}_{1}, \mathbf{X}_{2}, \mathbf{X}_{3}, \mathbf{X}_{4}\right\}$ as a subalgebra.

\section{Case 7}

Generators:

$$
\begin{aligned}
& \mathbf{X}_{1}=\partial_{t} \\
& \mathbf{X}_{2}=\partial_{y}, \\
& \mathbf{X}_{3}=\partial_{z}, \\
& \mathbf{X}_{4}=z \partial_{y}-y \partial_{z}, \\
& \mathbf{X}_{5}=\left(\frac{1}{\lambda T_{0}}-\frac{\lambda}{4} t^{2}\right) \partial_{t}+\frac{1}{\sqrt{T_{1}}} t \partial_{x}, \\
& \mathbf{X}_{6}=-\frac{\lambda}{2} t \partial_{t}+\frac{1}{\sqrt{T_{1}}} \partial_{x} .
\end{aligned}
$$


Algebra:

$$
\begin{array}{lll}
{\left[\mathbf{X}_{1}, \mathbf{X}_{5}\right]=\mathbf{X}_{6},} & {\left[\mathbf{X}_{1}, \mathbf{X}_{6}\right]=-\frac{\lambda}{2} \mathbf{X}_{1},} & {\left[\mathbf{X}_{2}, \mathbf{X}_{4}\right]=-\mathbf{X}_{3}} \\
{\left[\mathbf{X}_{3}, \mathbf{X}_{4}\right]=\mathbf{X}_{2},} & {\left[\mathbf{X}_{5}, \mathbf{X}_{6}\right]=\frac{\lambda}{2} \mathbf{X}_{5},} & {\left[\mathbf{X}_{i}, \mathbf{X}_{j}\right]=0, \text { otherwise }}
\end{array}
$$

This is a semisimple algebra having $\left\{\mathbf{X}_{1}, \mathbf{X}_{2}, \mathbf{X}_{3}, \mathbf{X}_{4}\right\}$ and $\left\{\mathbf{X}_{1}, \mathbf{X}_{5}, \mathbf{X}_{6}\right\}$ as subalgebras.

\section{Case 8}

Generators:

$$
\begin{aligned}
& \mathbf{X}_{1}=\partial_{t} \\
& \mathbf{X}_{2}=\partial_{y}, \\
& \mathbf{X}_{3}=\partial_{z}, \\
& \mathbf{X}_{4}=z \partial_{y}-y \partial_{z} .
\end{aligned}
$$

Algebra:

$$
\left[\mathbf{X}_{2}, \mathbf{X}_{4}\right]=-\mathbf{X}_{3}, \quad\left[\mathbf{X}_{3}, \mathbf{X}_{4}\right]=\mathbf{X}_{2}, \quad\left[\mathbf{X}_{i}, \mathbf{X}_{j}\right]=0 \text {, otherwise }
$$

This is the same as in Case 2 .

\section{Case 9}

Generators:

$$
\begin{aligned}
& \mathbf{X}_{1}=\partial_{t} \\
& \mathbf{X}_{2}=\partial_{y} \\
& \mathbf{X}_{3}=\partial_{z}, \\
& \mathbf{X}_{4}=z \partial_{y}-\frac{\beta}{\gamma} y \partial_{z}, \\
& \mathbf{X}_{5}=\frac{1}{\sqrt{T_{1}}} \partial_{x} \\
& \mathbf{X}_{6}=-\frac{1}{\alpha} \int \sqrt{T_{1}} d x \partial_{t}+\frac{1}{\sqrt{T_{1}}} t \partial_{x}, \\
& \mathbf{X}_{7}=y \partial_{t}+\frac{\alpha}{\beta} t \partial_{y}, \\
& \mathbf{X}_{8}=z \partial_{t}+\frac{\alpha}{\gamma} t \partial_{z}, \\
& \mathbf{X}_{9}=\frac{y}{\sqrt{T_{1}}} \partial_{x}-\frac{1}{\beta} \int \sqrt{T_{1}} d x \partial_{y}, \\
& \mathbf{X}_{10}=\frac{z}{\sqrt{T_{1}}} \partial_{x}-\frac{1}{\gamma} \int \sqrt{T_{1}} d x \partial_{z} .
\end{aligned}
$$


Algebra:

$$
\begin{array}{lll}
{\left[\mathbf{X}_{1}, \mathbf{X}_{6}\right]=\mathbf{X}_{5},} & {\left[\mathbf{X}_{1}, \mathbf{X}_{7}\right]=\frac{\alpha}{\beta} \mathbf{X}_{2},} & {\left[\mathbf{X}_{1}, \mathbf{X}_{8}\right]=\frac{\alpha}{\gamma} \mathbf{X}_{3},} \\
{\left[\mathbf{X}_{2}, \mathbf{X}_{4}\right]=-\frac{\beta}{\gamma} \mathbf{X}_{3},} & {\left[\mathbf{X}_{2}, \mathbf{X}_{7}\right]=\mathbf{X}_{1},} & {\left[\mathbf{X}_{2}, \mathbf{X}_{9}\right]=\mathbf{X}_{5},} \\
{\left[\mathbf{X}_{3}, \mathbf{X}_{4}\right]=\mathbf{X}_{2},} & {\left[\mathbf{X}_{3}, \mathbf{X}_{8}\right]=\mathbf{X}_{1},} & {\left[\mathbf{X}_{3}, \mathbf{X}_{10}\right]=\mathbf{X}_{5},} \\
{\left[\mathbf{X}_{4}, \mathbf{X}_{7}\right]=\mathbf{X}_{8},} & {\left[\mathbf{X}_{4}, \mathbf{X}_{8}\right]=-\frac{\beta}{\gamma} \mathbf{X}_{7},} & {\left[\mathbf{X}_{4}, \mathbf{X}_{9}\right]=\mathbf{X}_{10}} \\
{\left[\mathbf{X}_{4}, \mathbf{X}_{10}\right]=-\frac{\beta}{\gamma} \mathbf{X}_{9},} & {\left[\mathbf{X}_{5}, \mathbf{X}_{6}\right]=\frac{1}{\alpha} \mathbf{X}_{1},} & {\left[\mathbf{X}_{5}, \mathbf{X}_{9}\right]=-\frac{1}{\beta} \mathbf{X}_{2},} \\
{\left[\mathbf{X}_{5}, \mathbf{X}_{10}\right]=-\frac{1}{\gamma} \mathbf{X}_{3},} & {\left[\mathbf{X}_{6}, \mathbf{X}_{7}\right]=-\mathbf{X}_{9},} & {\left[\mathbf{X}_{6}, \mathbf{X}_{8}\right]=-\mathbf{X}_{10},} \\
{\left[\mathbf{X}_{6}, \mathbf{X}_{9}\right]=-\frac{1}{\alpha} \mathbf{X}_{7},} & {\left[\mathbf{X}_{6}, \mathbf{X}_{10}\right]=-\frac{1}{\alpha} \mathbf{X}_{8},} & {\left[\mathbf{X}_{7}, \mathbf{X}_{8}\right]=-\frac{\alpha}{\beta} \mathbf{X}_{4},} \\
{\left[\mathbf{X}_{7}, \mathbf{X}_{9}\right]=\frac{\alpha}{\beta} \mathbf{X}_{6},} & {\left[\mathbf{X}_{8}, \mathbf{X}_{10}\right]=\frac{\alpha}{\gamma} \mathbf{X}_{6},} & {\left[\mathbf{X}_{9}, \mathbf{X}_{10}\right]=\frac{1}{\beta} \mathbf{X}_{4},} \\
{\left[\mathbf{X}_{i}, \mathbf{X}_{j}\right]=0, \text { otherwise. }} &
\end{array}
$$

This semisimple algebra has a 3 dimensional subalgebra $\left\{\mathbf{X}_{4}, \mathbf{X}_{9}, \mathbf{X}_{10}\right\}$ of rotations, 4 dimensional subalgebra $\left\{\mathbf{X}_{1}, \mathbf{X}_{2}, \mathbf{X}_{3}, \mathbf{X}_{4}\right\}$ and 7 dimensional subalgebra $\left\{\mathbf{X}_{1}, \mathbf{X}_{2}, \mathbf{X}_{3}, \mathbf{X}_{4}, \mathbf{X}_{5}, \mathbf{X}_{9}, \mathbf{X}_{10}\right\}$ in it.

Case 10

Generators:

$$
\begin{aligned}
& \mathbf{X}_{1}=\partial_{t}, \\
& \mathbf{X}_{2}=\partial_{y}, \\
& \mathbf{X}_{3}=\partial_{z}, \\
& \mathbf{X}_{4}=z \partial_{y}-k y \partial_{z} .
\end{aligned}
$$

Algebra:

$$
\left[\mathbf{X}_{2}, \mathbf{X}_{4}\right]=-k \mathbf{X}_{3}, \quad\left[\mathbf{X}_{3}, \mathbf{X}_{4}\right]=\mathbf{X}_{2}, \quad\left[\mathbf{X}_{i}, \mathbf{X}_{j}\right]=0 \text {, otherwise }
$$

\section{Case 11}

Generators:

$$
\begin{aligned}
& \mathbf{X}_{1}=\partial_{t} \\
& \mathbf{X}_{2}=\partial_{y} \\
& \mathbf{X}_{3}=\partial_{z}, \\
& \mathbf{X}_{4}=z \partial_{y}-y \partial_{z} .
\end{aligned}
$$

Algebra:

$$
\left[\mathbf{X}_{2}, \mathbf{X}_{4}\right]=-\mathbf{X}_{3}, \quad\left[\mathbf{X}_{3}, \mathbf{X}_{4}\right]=\mathbf{X}_{2}, \quad\left[\mathbf{X}_{i}, \mathbf{X}_{j}\right]=0, \text { otherwise }
$$

Its structure is similar to that of Case 2. 


\section{Case 12}

Generators:

$$
\begin{aligned}
& \mathbf{X}_{1}=\partial_{t} \\
& \mathbf{X}_{2}=\partial_{y} \\
& \mathbf{X}_{3}=\partial_{z} \\
& \mathbf{X}_{4}=-z \partial_{y}+y \partial_{z} \\
& \mathbf{X}_{5}=\frac{1}{\sqrt{T_{1}}} \partial_{x}-k_{1} y \partial_{y}-k_{1} z \partial_{z} \\
& \mathbf{X}_{6}=\frac{y}{\sqrt{T_{1}}} \partial_{x}-\left(\int \frac{\sqrt{T_{1}}}{T_{2}} d x+k_{1} \frac{y^{2}}{2}-k_{1} \frac{z^{2}}{2}\right) \partial_{y}-k_{1} y z \partial_{z} \\
& \mathbf{X}_{7}=\frac{z}{\sqrt{T_{1}}} \partial_{x}-k_{1} y z \partial_{y}-\left(\int \frac{\sqrt{T_{1}}}{T_{2}} d x-\frac{k_{1}}{2 d} y^{2}+k_{1} \frac{z^{2}}{2}\right) \partial_{z}
\end{aligned}
$$

Algebra:

$$
\begin{array}{lll}
{\left[\mathbf{X}_{2}, \mathbf{X}_{4}\right]=\mathbf{X}_{3},} & {\left[\mathbf{X}_{2}, \mathbf{X}_{5}\right]=-k_{1} \mathbf{X}_{2},} & {\left[\mathbf{X}_{2}, \mathbf{X}_{6}\right]=\mathbf{X}_{5},} \\
{\left[\mathbf{X}_{2}, \mathbf{X}_{7}\right]=k_{1} \mathbf{X}_{4},} & {\left[\mathbf{X}_{3}, \mathbf{X}_{4}\right]=-\mathbf{X}_{2},} & {\left[\mathbf{X}_{3}, \mathbf{X}_{5}\right]=-k_{1} \mathbf{X}_{3},} \\
{\left[\mathbf{X}_{3}, \mathbf{X}_{6}\right]=-k_{1} \mathbf{X}_{4},} & {\left[\mathbf{X}_{3}, \mathbf{X}_{7}\right]=\mathbf{X}_{5},} & {\left[\mathbf{X}_{4}, \mathbf{X}_{6}\right]=-\mathbf{X}_{7},} \\
{\left[\mathbf{X}_{4}, \mathbf{X}_{7}\right]=-\mathbf{X}_{6},} & {\left[\mathbf{X}_{5}, \mathbf{X}_{6}\right]=-\mathbf{X}_{6},} & {\left[\mathbf{X}_{5}, \mathbf{X}_{7}\right]=-\mathbf{X}_{7},} \\
{\left[\mathbf{X}_{6}, \mathbf{X}_{7}\right]=K \mathbf{X}_{4},} & {\left[\mathbf{X}_{i}, \mathbf{X}_{j}\right]=0, \text { otherwise. }} &
\end{array}
$$

where $K=-2 k_{1}\left(\int \frac{\sqrt{T_{1}}}{T_{2}} d x+\frac{1}{2 k_{1} T_{2}}\right)$ is a constant. This is a semisimple algebra having $\left\{\mathbf{X}_{1}, \mathbf{X}_{2}, \mathbf{X}_{3}, \mathbf{X}_{4}\right\}$ and $\left\{\mathbf{X}_{4}, \mathbf{X}_{5}, \mathbf{X}_{6}, \mathbf{X}_{7}\right\}$ as 4 dimensional subalgebras and a 6 dimensional subalgebra $\left\{\mathbf{X}_{2}, \mathbf{X}_{3}, \mathbf{X}_{4}, \mathbf{X}_{5}, \mathbf{X}_{6}, \mathbf{X}_{7}\right\}$ in it. We write this as $G_{7}=$ $\left\langle G_{4}, \mathbf{X}_{5}, \mathbf{X}_{6}, \mathbf{X}_{7}\right\rangle$ where $G_{4}=\{S O(2) \times[\mathbb{R} \otimes S O(2)]\} \otimes \mathbb{R}$.

Case 13

Generators:

Algebra:

$$
\begin{aligned}
& \mathbf{X}_{1}=\partial_{t} \\
& \mathbf{X}_{2}=\partial_{y} \\
& \mathbf{X}_{3}=\partial_{z} \\
& \mathbf{X}_{4}=z \partial_{y}-y \partial_{z}, \\
& \mathbf{X}_{5}=t \partial_{t}-\frac{2 T_{0}}{T_{0}^{\prime}} \partial_{x}+\frac{1}{k_{2}} y \partial_{y}+\frac{1}{k_{2}} z \partial_{z} .
\end{aligned}
$$

$$
\begin{array}{lll}
{\left[\mathbf{X}_{1}, \mathbf{X}_{5}\right]=\mathbf{X}_{1},} & {\left[\mathbf{X}_{2}, \mathbf{X}_{4}\right]=-\mathbf{X}_{3},} & {\left[\mathbf{X}_{2}, \mathbf{X}_{5}\right]=\frac{1}{k_{2}} \mathbf{X}_{2}} \\
{\left[\mathbf{X}_{3}, \mathbf{X}_{4}\right]=\mathbf{X}_{2},} & {\left[\mathbf{X}_{3}, \mathbf{X}_{5}\right]=\frac{1}{k_{2}} \mathbf{X}_{3},} & {\left[\mathbf{X}_{i}, \mathbf{X}_{j}\right]=0, \text { otherwise. }}
\end{array}
$$

This is a solvable algebra which can be written as $G=\left\langle G_{4}, \mathbf{X}_{5}\right\rangle$, where $G_{4}=\{S O(2) \times[\mathbb{R} \otimes S O(2)]\} \otimes \mathbb{R}$. 


\section{Case 14}

Generators:

$$
\begin{aligned}
& \mathbf{X}_{1}=\partial_{t} \\
& \mathbf{X}_{2}=\partial_{y} \\
& \mathbf{X}_{3}=\partial_{z} \\
& \mathbf{X}_{4}=-z \partial_{y}+y \partial_{z} \\
& \mathbf{X}_{5}=y \partial_{t}+\alpha t \partial_{y} \\
& \mathbf{X}_{6}=z \partial_{t}+\alpha t \partial_{z} \\
& \mathbf{X}_{7}=\left(\frac{y^{2}}{2}+\frac{z^{2}}{2}+\alpha \frac{t^{2}}{2}\right) \partial_{t}-\frac{2 \alpha T_{0}}{T_{0}^{\prime}} t \partial_{x}+\alpha t y \partial_{y}+\alpha t z \partial_{z} \\
& \mathbf{X}_{8}=t y \partial_{t}-\frac{2 T_{0}}{T_{0}^{\prime}} y \partial_{x}+\left(\alpha \frac{t^{2}}{2}+\frac{y^{2}}{2}-\frac{z^{2}}{2}\right) \partial_{y}+y z \partial_{z}, \\
& \mathbf{X}_{9}=t z \partial_{t}-\frac{2 T_{0}}{T_{0}^{\prime}} z \partial_{x}+y z \partial_{y}+\left(\alpha \frac{t^{2}}{2}-\frac{y^{2}}{2}+\frac{z^{2}}{2}\right) \partial_{z}, \\
& \mathbf{X}_{10}=t \partial_{t}-\frac{2 T_{0}}{T_{0}^{\prime}} \partial_{x}+y \partial_{y}+z \partial_{z} . \\
&
\end{aligned}
$$

Algebra:

$$
\begin{array}{lll}
{\left[\mathbf{X}_{1}, \mathbf{X}_{5}\right]=\alpha \mathbf{X}_{2},} & {\left[\mathbf{X}_{1}, \mathbf{X}_{6}\right]=\alpha \mathbf{X}_{3},} & {\left[\mathbf{X}_{1}, \mathbf{X}_{7}\right]=\alpha \mathbf{X}_{10},} \\
{\left[\mathbf{X}_{1}, \mathbf{X}_{8}\right]=\mathbf{X}_{5},} & {\left[\mathbf{X}_{1}, \mathbf{X}_{9}\right]=\mathbf{X}_{6},} & {\left[\mathbf{X}_{1}, \mathbf{X}_{10}\right]=\mathbf{X}_{1},} \\
{\left[\mathbf{X}_{2}, \mathbf{X}_{4}\right]=\mathbf{X}_{3},} & {\left[\mathbf{X}_{2}, \mathbf{X}_{5}\right]=\mathbf{X}_{1},} & {\left[\mathbf{X}_{2}, \mathbf{X}_{7}\right]=\mathbf{X}_{5},} \\
{\left[\mathbf{X}_{2}, \mathbf{X}_{8}\right]=\mathbf{X}_{10},} & {\left[\mathbf{X}_{2}, \mathbf{X}_{9}\right]=-\mathbf{X}_{4},} & {\left[\mathbf{X}_{2}, \mathbf{X}_{10}\right]=\mathbf{X}_{2},} \\
{\left[\mathbf{X}_{3}, \mathbf{X}_{4}\right]=-\mathbf{X}_{2},} & {\left[\mathbf{X}_{3}, \mathbf{X}_{6}\right]=\mathbf{X}_{1},} & {\left[\mathbf{X}_{3}, \mathbf{X}_{7}\right]=\mathbf{X}_{6},} \\
{\left[\mathbf{X}_{3}, \mathbf{X}_{8}\right]=\mathbf{X}_{4},} & {\left[\mathbf{X}_{3}, \mathbf{X}_{9}\right]=\mathbf{X}_{10},} & {\left[\mathbf{X}_{3}, \mathbf{X}_{10}\right]=\mathbf{X}_{3},} \\
{\left[\mathbf{X}_{4}, \mathbf{X}_{5}\right]=-\mathbf{X}_{6},} & {\left[\mathbf{X}_{4}, \mathbf{X}_{6}\right]=\mathbf{X}_{5},} & {\left[\mathbf{X}_{4}, \mathbf{X}_{8}\right]=-\mathbf{X}_{9},} \\
{\left[\mathbf{X}_{4}, \mathbf{X}_{9}\right]=\mathbf{X}_{8},} & {\left[\mathbf{X}_{5}, \mathbf{X}_{6}\right]=\alpha \mathbf{X}_{4},} & {\left[\mathbf{X}_{5}, \mathbf{X}_{7}\right]=\alpha \mathbf{X}_{8},} \\
{\left[\mathbf{X}_{5}, \mathbf{X}_{8}\right]=\mathbf{X}_{7},} & {\left[\mathbf{X}_{6}, \mathbf{X}_{7}\right]=-\mathbf{X}_{9},} & {\left[\mathbf{X}_{6}, \mathbf{X}_{9}\right]=\mathbf{X}_{7},} \\
{\left[\mathbf{X}_{7}, \mathbf{X}_{10}\right]=-\mathbf{X}_{7},} & {\left[\mathbf{X}_{8}, \mathbf{X}_{10}\right]=-\mathbf{X}_{8},} & {\left[\mathbf{X}_{9}, \mathbf{X}_{10}\right]=-\mathbf{X}_{9},} \\
{\left[\mathbf{X}_{i}, \mathbf{X}_{j}\right]=0, \text { otherwise. }} &
\end{array}
$$

It has 3 dimensional subalgebras $\left\{\mathbf{X}_{4}, \mathbf{X}_{5}, \mathbf{X}_{6}\right\}$ of rotations and $\left\{\mathbf{X}_{8}, \mathbf{X}_{9}, \mathbf{X}_{10}\right\} ; 4$ dimensional subalgebras $\left\{\mathbf{X}_{1}, \mathbf{X}_{2}, \mathbf{X}_{3}, \mathbf{X}_{4}\right\}$ and $\left\{\mathbf{X}_{7}, \mathbf{X}_{8}, \mathbf{X}_{9}, \mathbf{X}_{10}\right\}$; and 6 dimensional subalgebras $\left\{\mathbf{X}_{1}, \mathbf{X}_{2}, \mathbf{X}_{3}, \mathbf{X}_{4}, \mathbf{X}_{5}, \mathbf{X}_{6}\right\}$ in it. This $S O(1,4)$ or $S O(2,3)$ anti-de Sitter Lie algebra is the maximal semisimple algebra of the degenerate case.

\section{Examples of metrics}

Here we give a few examples of spacetimes comparing MCs with their RCs $[4,5]$ and $\mathrm{KVs}[8]$. 
1.

$$
d s^{2}=e^{\nu}\left(d t^{2}-d y^{2}-d z^{2}\right)-d x^{2},\left(\nu^{\prime \prime} \neq 0\right)
$$

For this metric the non-vanishing components of $T_{a b}$ are

$$
\begin{aligned}
& T_{00}=-\frac{e^{\nu}}{4}\left(4 \nu^{\prime \prime}+3 \nu^{\prime^{2}}\right) \\
& T_{11}=\frac{1}{4}\left(3 \mu^{\prime 2}\right), \\
& T_{22}=\frac{e^{\nu}}{4}\left(4 \nu^{\prime \prime}+3 \nu^{\prime 2}\right)=T_{33},
\end{aligned}
$$

and those of the Ricci tensor are

$$
\begin{aligned}
& R_{00}=\frac{e^{\nu}}{4}\left(2 \nu^{\prime \prime}+3 \nu^{\prime 2}\right) \\
& R_{11}=-3\left(\frac{\nu^{\prime \prime}}{2}+\frac{\nu^{\prime 2}}{4}\right), \\
& R_{22}=-\frac{e^{\nu}}{4}\left(2 \mu^{\prime \prime}+3 \nu^{\prime 2}\right)=R_{33} .
\end{aligned}
$$

It has $6 \mathrm{KVs}, 6 \mathrm{RCs}$ and $6 \mathrm{MCs}$ given in Case 1.

2.

$$
d s^{2}=e^{\nu} d t^{2}-d x^{2}-e^{\mu}\left(d y^{2}+d z^{2}\right),\left(\nu^{\prime \prime} \neq 0, \mu^{\prime \prime} \neq 0\right) .
$$

It admits $4 \mathrm{KVs}, 4 \mathrm{RCs}$ and 4 MCs given by Case 2 .

3.

$$
d s^{2}=e^{A x}\left(d t^{2}-d y^{2}-d z^{2}\right)-d x^{2}
$$

This is an anti-de Sitter metric admitting $10 \mathrm{KVs}, 10 \mathrm{RCs}$ and $10 \mathrm{MCs}$ given by Case 5 .

4.

$$
d s^{2}=x^{a} d t^{2}-d x^{2}-x^{b}\left(d y^{2}+d z^{2}\right) .
$$

The $T_{a b}$ in this case are

$$
\begin{aligned}
& T_{00}=\left(a-\frac{3}{4} a^{2}\right) x^{c-2}, \\
& T_{11}=\left(\frac{c a}{2}+\frac{a^{2}}{4}\right) / x^{2}, \\
& T_{22}=\frac{1}{4}\left(a^{2}+c^{2}-2 c-2 a+a c\right) x^{a-2}=T_{33} .
\end{aligned}
$$

This is an example of Case 4 with $4 \mathrm{KVs}, 5 \mathrm{RCs}$ and $5 \mathrm{MCs}$. 
5.

$$
d s^{2}=\left(x / x_{0}\right)^{2 a} d t^{2}-d x^{2}-\left(x / x_{0}\right)^{2}\left(d y^{2}+d z^{2}\right)
$$

$a$ and $x_{0}$ are constants and $a \neq 0,1,-1$. For this metric $R_{a b}$ are given by

$$
\begin{aligned}
& R_{00}=a(1+a) x^{2 a-2} / x_{0}^{2 a}, \\
& R_{11}=-\left(-a+a^{2}\right) / x^{2}, \\
& R_{22}=-(1+a) / x_{0}^{2}=R_{33} .
\end{aligned}
$$

The energy-momentum tensor for this metric can be written as with

$$
\begin{aligned}
& T_{00}=-x^{2 a-2} / x_{0}^{2 a}, \\
& T_{11}=(2 a+1) / x^{2}, \\
& T_{22}=a^{2} / x_{0}^{2}=T_{33} .
\end{aligned}
$$

It has $4 \mathrm{KVs}, 6 \mathrm{RCs}$ and $6 \mathrm{MCs}$ (Case 7). The energy density is negative and cannot be made positive by introducing a cosmological constant, therefore, it is unphysical.

6.

$$
d s^{2}=d t^{2}-d x^{2}-d y^{2}-d z^{2}
$$

For this metric $T_{a b}=R_{a b}=0$, for all $a, b$, which means that every direction is an MC and similarly RCs are also arbitrary functions of the coordinates $t$, $x, y$ and $z$ giving an infinite dimensional Lie algebra. However, it has $10 \mathrm{KVs}$. This is a wrapped Minkowski spacetime.

7 .

$$
d s^{2}=d t^{2}-d x^{2}-e^{A x}\left(d y^{2}-d z^{2}\right)
$$

$A$ is a non-zero constant. For this metric the non-vanishing components of $T_{a b}$ are

$$
\begin{aligned}
& T_{00}=-\frac{3 A^{2}}{4}, \\
& T_{11}=\frac{A^{2}}{4}, \\
& T_{22}=\frac{A^{2} e^{A x}}{4}=T_{33},
\end{aligned}
$$

and those of the Ricci tensor are

$$
\begin{aligned}
& R_{00}=0 \\
& R_{11}=-\frac{A^{2}}{2}, \\
& R_{22}=-\frac{A^{2} e^{A x}}{2}=R_{33} .
\end{aligned}
$$

It admits $7 \mathrm{KVs}$, infinitely many RCs and $7 \mathrm{MCs}$, given in Case 12 . 
8.

$$
d s^{2}=e^{A x} d t^{2}-d x^{2}-e^{B x}\left(d y^{2}+d z^{2}\right)
$$

For this metric the non-vanishing components of $T_{a b}$ are

$$
\begin{aligned}
& T_{00}=-\frac{3 B^{2} e^{A x}}{4} \\
& T_{11}=\frac{1}{4}\left(2 A B+B^{2}\right) \\
& T_{22}=\frac{e^{B x}}{4}\left(A^{2}+B^{2}+A B\right)=T_{33},
\end{aligned}
$$

and those of the Ricci tensor are

$$
\begin{aligned}
& R_{00}=\frac{e^{A x}}{4}\left(A^{2}+2 A B\right), \\
& R_{11}=-\left(\frac{A^{2}}{4}+\frac{B^{2}}{2}\right) \\
& R_{22}=-\frac{e^{B x}}{4}\left(A B+2 B^{2}\right)=R_{33} .
\end{aligned}
$$

Now, if $A \neq B$ it admits $5 \mathrm{KVs}, 5 \mathrm{RCs}$ and $5 \mathrm{MCs}$, otherwise it has $10 \mathrm{RCs}$ and 10 MCs.

9 .

$$
d s^{2}=\left(x / x_{0}\right)^{2 a} d t^{2}-d x^{2}-\left(x / x_{0}\right)^{4 / 3}\left(d y^{2}+d z^{2}\right)
$$

$a$ and $x_{0}$ are constants and $a \neq 0,1,-1$. The energy-momentum tensor for this metric can be written as with

$$
\begin{aligned}
& T_{00}=0 \\
& T_{11}=16 / 9 x^{2} \\
& T_{22}=-\left(a^{2}-\frac{a}{3}-\frac{2}{9}\right) / x^{2 / 3} x_{0}^{4 / 3}=T_{33} .
\end{aligned}
$$

For this metric $R_{a b}$ are given by

$$
\begin{aligned}
& R_{00}=a\left(a+\frac{1}{3}\right) x^{2 a-2} / x_{0}^{2 a}, \\
& R_{11}=\left(a-a^{2}-\frac{4}{3}\right) / x^{2} \\
& R_{22}=-\frac{2}{3}(a+3) / x^{2 / 3} x_{0}^{4 / 3}=R_{33} .
\end{aligned}
$$

This space admits $4 \mathrm{KVs}, 5 \mathrm{RCs}$ and infinitely many MCs (Case 15). 


\section{Conclusion}

The plane symmetric static spacetimes have been studied for their MCs. The MC equations have been solved giving rise to various cases characterized by the constraints on the components of the energy-momentum tensor. This includes cases of the non-degenerate as well the degenerate tensor. Their Lie algebra structure has also been given. Particular examples of metrics have been provided for which MCs have been compared with their KVs and RCs. In some spaces the RCs are greater than the MCs, while in others the MCs are more than the RCs, which shows that neither of the sets contains the other, in general.

\section{Acknowledgments}

The author is grateful to Asghar Qadir for reading the manuscript and suggesting some improvements. He would also like to thank M. Ziad, U. Camci and M. Sharif for useful discussions.

\section{References}

[1] Eisenhart, L. P., Continuous Groups of Transformations (Princeton University Press) 1933.

Petrov, A. Z., Einstein Spaces, (Pergamon Press) 1969;

Katzin, G. H., Levine, J., and Davis, W. R., J. Math. Phys. 10 (1969) 617;

Katzin, G. H., Levine, J., and Davis, W. R., J. Math. Phys. 11 (1970) 1518.

[2] Qadir A., Spacetime symmetries and their significance, in Applications of Symmetry Methods, Eds. Qadir A. and Saifullah K., (National Centre for Physics, Islamabad) 2006.

[3] Hall, G. S., Roy, I., and Vaz, E. G. L. R., Gen. Rel. Grav. 28 (1996) 299;

Camci, U., and Sharif. M., Gen. Rel. Grav. 35 (2003) 97;

Camci, U., and Sharif. M., Matter Collineations of Some Well-known Rotating Metrics, in Applications of Symmetry Methods, Eds. Qadir A. and Saifullah K., (National Centre for Physics, Islamabad) 2006;

Duggal, K. L., and Sharma, R., Symmetries of Spacetimes and Riemannian Manifolds, (Kluwer Academic Publishers) 1999;

Khan, H., M.Phil. Dissertation, Quaid-i-Azam University, Islamabad, 2003. 
[4] Qadir, A., Saifullah, K., and Ziad, M., Gen. Rel. Grav. 35 (2003) 1927;

Saifullah, K., Ph.D. Thesis, Quaid-i-Azam University, Islamabad, 2003.

[5] Farid, T. B., Qadir, A., and Ziad, M., J. Math. Phys. 36 (1995) 5812.

[6] Saifullah, K., Nuovo Cimento B 118 (2003) 1137 (Special issue devoted to Proc.

Xth ICRA Network Workshop "Black Holes, Gravitational Waves and Cosmology", Rome, Pescara, 2002).

[7] Qadir, A., personal communication, on the relationship of matter collineations with Ricci collineations.

[8] Qadir, A., and Ziad, M., Proceedings of the 6th Marcel Grossmann Meeting, edited by Nakamura, T., and Sato, H., (World Scientific), 1993, p. 1115.

[9] Kramer, D., Stephani, H., MacCallum, M. A. H., and Herlt, E., Exact Solutions of Einstein's Field Equations (Cambridge University Press,) 1980.

[10] Bokhari, A. H., and Qadir, A., J. Math. Phys. 28 (1987) 1019;

Bokhari, A. H., and Qadir, A., J. Math. Phys. 29 (1988) 525;

Qadir, A., and Ziad, M., J. Math. Phys. 29 (1988) 2473;

Qadir, A., and Ziad, M., J. Math. Phys. 31 (1990) 254;

Ziad, M., Ph.D. Thesis, Quaid-i-Azam University, Islamabad, 1990. 